\title{
A VORONOVSKAYA-TYPE THEOREM FOR A POSITIVE LINEAR OPERATOR
}

\author{
ALEXANDRA CIUPA
}

Received 23 March 2005; Revised 20 December 2005; Accepted 4 January 2006

We consider a sequence of positive linear operators which approximates continuous functions having exponential growth at infinity. For these operators, we give a Voronovskayatype theorem.

Copyright (c) 2006 Hindawi Publishing Corporation. All rights reserved.

\section{Introduction}

Sequences of positive linear operators are often used in approximation theory. Let $\left(L_{n}\right)_{n \geq 1}$ be such a sequence, where the operators $L_{n}$ are defined on a suitable linear subspace $E$ of $C(I), I \subset \mathbb{R}$ an interval. An important problem is the investigation of the limit

$$
\lim _{n \rightarrow \infty} n\left(L_{n} f-f\right)
$$

in order to obtain information about the rate of convergence and the saturation properties of the sequence $\left(L_{n}\right)$.

The above formula is called Voronovskaya's formula for the sequence $\left(L_{n}\right)_{n \geq 1}$.

This paper is devoted to establishing a Voronovskaya-type formula for the sequence of positive linear operators introduced in [1], which approximate continuous functions of exponential order. To obtain the operators, we consider $g(z)=\sum_{n=0}^{\infty} a_{n} z^{n}, g(1) \neq 0$, an analytic function in the disk $|z|<R, R>1$, and we define the polynomials $p_{k}$ by the relation

$$
g(u) \cosh (u x)=\sum_{k=0}^{\infty} p_{k}(x) u^{k},
$$

where $\cosh x=\sum_{k=0}^{\infty}\left(x^{2 k} /(2 k)\right.$ !) is the hyperbolic cosine of $x$. Therefore, the polynomials are

$$
p_{k}(x)=\sum_{\nu=0}^{k} a_{\nu} \frac{x^{k-\nu}}{(k-v) !} \cdot \frac{1+(-1)^{k-\nu}}{2} .
$$


2 A Voronovskaya-type theorem for a positive linear operator

Let $C[0, \infty)$ be the set of all real-valued functions continuous on $[0, \infty)$ and $w_{p}(x)=$ $e^{-p x}, x \geq 0, p>0$, the weight function. We will work in the space of functions $C_{p}=\{f \in$ $C[0, \infty): w_{p} f$ is uniformly continuous and bounded on $\left.[0, \infty)\right\}$, with the norm $\|f\|_{p}=$ $\sup _{x \in[0, \infty)} w_{p}(x)|f(x)|$.

We define the operator $P_{n}: C_{p} \rightarrow C_{r}, r>p$, by the relation

$$
P_{n}(f ; x)=\frac{1}{g(1) \cosh (n x)} \sum_{k=0}^{\infty} p_{k}(n x) f\left(\frac{k}{n}\right) .
$$

We consider that $a_{n} / g(1) \geq 0, n=0,1, \ldots$, which implies that the $P_{n}$ operator is positive.

We proved in [1] the following theorem.

Theorem 1.1. If $f \in C_{p}$, then for each $x \geq 0, \lim _{n \rightarrow \infty} P_{n}(f ; x)=f(x)$, the convergence being uniform in each interval $[0, a]$.

Remark 1.2. (1) If in (1.2) we consider $g(u)=\cosh u$, the operator $P_{n}$ becomes

$$
L_{n}(f ; x)=\frac{1}{\cosh 1 \cosh (n x)} \sum_{k=0}^{\infty} p_{2 k}(n x) f\left(\frac{2 k}{n}\right) \text {, }
$$

where

$$
p_{2 k}(x)=\frac{(1+x)^{2 k}+(1-x)^{2 k}}{2(2 k) !}
$$

which was studied in [2].

(2) If instead of (1.2) we consider the relation

$$
\cosh (u x)=\sum_{k=0}^{\infty} p_{k}(x) u^{k}
$$

we obtain

$$
p_{2 k}(x)=\frac{x^{2 k}}{(2 k) !}
$$

The operator

$$
L_{n}^{*}(f ; x)=\frac{1}{\cosh (n x)} \sum_{k=0}^{\infty} \frac{(n x)^{2 k}}{(2 k) !} f\left(\frac{2 k}{n}\right)
$$

was studied by Leśniewicz and Rempulska [3].

\section{Auxiliary results}

In order to prove a Voronovskaya-type theorem, we need some auxiliary results. 
Lemma 2.1. For $x \in[0, \infty)$ and $n \in \mathbb{N}$,

$$
\begin{gathered}
P_{n}\left(e_{0} ; x\right)=1, \\
P_{n}\left(e_{1} ; x\right)=x \tanh (n x)+\frac{1}{n} \cdot \frac{g^{\prime}(1)}{g(1)} \\
P_{n}\left(e_{2} ; x\right)=x^{2}+\frac{x}{n} \tanh (n x) \frac{2 g^{\prime}(1)+g(1)}{g(1)}+\frac{1}{n^{2}} \cdot \frac{g^{\prime \prime}(1)+g^{\prime}(1)}{g(1)},
\end{gathered}
$$

where $e_{i}(x)=x^{i}, i \in\{0,1,2\}$, and $\tanh x$ is the hyperbolic tangent of $x$.

Lemma 2.2. For $x \in[0, \infty)$ and $n \in \mathbb{N}$, the following hold:

$$
\begin{gathered}
P_{n}(t-x ; x)=-x(1-\tanh (n x))+\frac{1}{n} \cdot \frac{g^{\prime}(1)}{g(1)}, \\
P_{n}\left((t-x)^{2} ; x\right)=(1-\tanh (n x))\left[2 x^{2}-\frac{x}{n}\left(1+\frac{2 g^{\prime}(1)}{g(1)}\right)\right]+\frac{x}{n}+\frac{1}{n^{2}} \cdot \frac{g^{\prime \prime}(1)+g^{\prime}(1)}{g(1)}, \\
P_{n}\left((t-x)^{4} ; x\right)=(1-\tanh (n x))\left(a_{1} x^{4}-a_{2} \frac{x^{3}}{n}+a_{3} \frac{x^{2}}{n^{2}}-a_{4} \frac{x}{n^{3}}\right)+a_{5} \frac{x^{2}}{n^{2}}+a_{6} \frac{x}{n^{3}}+a_{7} \frac{1}{n^{4}},
\end{gathered}
$$

where $a_{i}, i=\overline{1,7}$, are positive constants:

$$
\begin{gathered}
a_{1}=8, \quad a_{2}=12+\frac{16 g^{\prime}(1)}{g(1)}, \quad a_{3}=4\left(1+\frac{6 g^{\prime}(1)+3 g^{\prime \prime}(1)}{g(1)}\right), \\
a_{4}=1+\frac{14 g^{\prime}(1)+18 g^{\prime \prime}(1)+4 g^{(3)}(1)}{g(1)}, \quad a_{5}=3, \\
a_{6}=1+\frac{6 g^{\prime \prime}(1)+10 g^{\prime}(1)}{g(1)}, \quad a_{7}=\frac{g^{\prime}(1)+7 g^{\prime \prime}(1)+6 g^{(3)}(1)+g^{(4)}(1)}{g(1)} .
\end{gathered}
$$

Lemmas 2.1 and 2.2 can be proved by means of successive partial differentiation with respect to $u$ in the generating relation (1.2), and putting then $u=1$.

Lemma 2.3. For every fixed point $x_{0} \in[0, \infty)$,

$$
\lim _{n \rightarrow \infty} n P_{n}\left(t-x_{0} ; x_{0}\right)=\frac{g^{\prime}(1)}{g(1)}, \quad \lim _{n \rightarrow \infty} n P_{n}\left(\left(t-x_{0}\right)^{2} ; x_{0}\right)=x_{0} .
$$

Proof. Because $1-\tanh (n x)=2 /\left(e^{2 n x}+1\right)$, by Lemma 2.2 we have

$$
\begin{gathered}
n P_{n}\left(t-x_{0} ; x_{0}\right)=\frac{-2 n x_{0}}{e^{2 n x_{0}}+1}+\frac{g^{\prime}(1)}{g(1)} \\
n P_{n}\left(\left(t-x_{0}\right)^{2} ; x_{0}\right)=\frac{2 n x_{0}}{e^{2 n x_{0}}+1}\left[2 x_{0}^{2}-\frac{x_{0}}{n}\left(1+2 \frac{g^{\prime}(1)}{g(1)}\right)\right]+x_{0}+\frac{1}{n} \cdot \frac{g^{\prime \prime}(1)+g^{\prime}(1)}{g(1)} .
\end{gathered}
$$

Therefore Lemma 2.3 holds. 
4 A Voronovskaya-type theorem for a positive linear operator

Lemma 2.4. For each fixed point $x_{0} \in[0, \infty)$, there is a positive constant $M_{1}\left(x_{0}\right)$, depending only on $x_{0}$ such that

$$
P_{n}\left(\left(t-x_{0}\right)^{4} ; x_{0}\right) \leq M_{1}\left(x_{0}\right) \frac{1}{n^{2}}
$$

for all $n \in \mathbb{N}$.

Proof. For $x \geq 0$ and $r, n \in \mathbb{N}$, we have

$$
x^{r}(1-\tanh (n x)) \leq \frac{2^{1-r}}{n^{r}} r !
$$

By Lemma 2.2, it results that

$$
\begin{aligned}
P_{n}\left(\left(t-x_{0}\right)^{4} ; x_{0}\right) \leq & a_{1} \frac{2^{-3}}{n^{4}} 4 !-a_{2} \frac{2^{-2}}{n^{4}} 3 !+f_{3} \frac{2^{-1}}{n^{4}} 2 ! \\
& -a_{4} \frac{1}{n^{4}}+a_{5} \frac{x_{0}^{2}}{n^{2}}+a_{6} \frac{x_{0}}{n^{3}}+a_{7} \frac{1}{n^{4}} \leq M_{1}\left(x_{0}\right) \frac{1}{n^{2}}
\end{aligned}
$$

We proved in [1] the following lemma.

LEMMA 2.5. Let $p>0$, let $r>p$, and let $n_{0}$ be a natural number such that $n_{0}>p /(\ln r-\ln p)$. Then there exists a positive constant $M_{p, r}$ depending only on $p$ and $r$ such that

$$
e^{-r x}\left(P_{n}(t-x)^{2} e^{p t} ; x\right) \leq M_{p, r} \frac{x+1}{n}
$$

for all $x \geq 0$ and $n \geq n_{0}$.

Lemma 2.6. Let $x_{0} \in[0, \infty)$ be a fixed point and $\varphi\left(\cdot ; x_{0}\right) \in C_{p}$ a function such that

$$
\lim _{t \rightarrow x_{0}} \varphi\left(t ; x_{0}\right)=0
$$

Then

$$
\lim _{n \rightarrow \infty} P_{n}\left(\varphi\left(t ; x_{0}\right) ; x_{0}\right)=0
$$

Proof. Let $r>p>0$. For every fixed $x_{0} \geq 0$ and $n \in \mathbb{N}$, we have

$$
e^{-r x_{0}} P_{n}\left(\varphi\left(t ; x_{0}\right) ; x_{0}\right)=\frac{e^{-r x_{0}}}{g(1) \cosh (n x)} \sum_{k=0}^{\infty} p_{k}\left(n x_{0}\right) \varphi\left(\frac{k}{n} ; x_{0}\right) .
$$

By the properties of function $\varphi\left(\cdot ; x_{0}\right)$, it results that for all $\varepsilon>0$ there exists a positive constant $\delta(\varepsilon)$ such that if $\left|t-x_{0}\right|<\delta$, then $\left|\varphi\left(t ; x_{0}\right)\right|<\varepsilon / 2, t \geq 0$. Moreover, there exists a positive constant $M_{2} \equiv M_{2}(p)$ such that

$$
e^{-p t}\left|\varphi\left(t ; x_{0}\right)\right| \leq M_{2} \quad \forall t \geq 0
$$


Now we can write

$$
\begin{aligned}
e^{-r x_{0}} P_{n}\left(\varphi\left(t ; x_{0}\right) ; x_{0}\right) \leq & \frac{e^{-r x_{0}}}{g(1) \cosh \left(n x_{0}\right)} \sum_{\left|k / n-x_{0}\right|<\delta} p_{k}\left(\left(n x_{0}\right)\right)\left|\varphi\left(\frac{k}{n} ; x_{0}\right)\right| \\
& +\frac{e^{-r x_{0}}}{g(1) \cosh n x_{0}} \sum_{\left|k / n-x_{0}\right| \geq \delta} p_{k}\left(n x_{0}\right)\left|\varphi\left(\frac{k}{n} ; x_{0}\right)\right|:=S_{1}+S_{2} .
\end{aligned}
$$

By the above properties of function $\varphi\left(\cdot ; x_{0}\right)$, it follows that

$$
\begin{aligned}
S_{1} & <\frac{\varepsilon}{2} \cdot \frac{e^{-r x_{0}}}{g(1) \cosh \left(n x_{0}\right)} \sum_{\left|k / n-x_{0}\right|<\delta} p_{k}\left(n x_{0}\right)<\frac{\varepsilon}{2} e^{-r x_{0}} P_{n}\left(1, x_{0}\right)<\frac{\varepsilon}{2}, \\
S_{2} & =\frac{e^{-r x_{0}}}{g(1) \cosh \left(n x_{0}\right)} \sum_{\left|k / n-x_{0}\right| \geq \delta} p_{k}\left(\left(n x_{0}\right)\right)\left|\varphi\left(\frac{k}{n} ; x_{0}\right)\right| e^{-p k / n} e^{p k / n} \\
& \leq M_{2} \frac{e^{-r x_{0}}}{g(1) \cosh n x_{0}} \sum_{\left|k / n-x_{0}\right| \geq \delta} p_{k}\left(n x_{0}\right) e^{p k / n} .
\end{aligned}
$$

But if

$$
\left|\frac{k}{n}-x_{0}\right| \geq \delta
$$

then

$$
1 \leq \frac{1}{\delta^{2}}\left(\frac{k}{n}-x_{0}\right)^{2}
$$

and by Lemma 2.5, we can write

$$
\begin{aligned}
S_{2} & \leq M_{2} \frac{1}{\delta^{2}} \cdot \frac{e^{-r x_{0}}}{g(1) \cosh \left(n x_{0}\right)} \sum_{\left|k / n-x_{0}\right| \geq \delta} p_{k}\left(n x_{0}\right)\left(\frac{k}{n}-x_{0}\right)^{2} e^{p k / n} \\
& \leq M_{2} \frac{e^{-r x_{0}}}{\delta^{2}} P_{n}\left(\left(t-x_{0}\right)^{2} e^{p t} ; x_{0}\right) \leq M_{2} \frac{1}{\delta^{2}} M_{p, r} \frac{x_{0}+1}{n}
\end{aligned}
$$

for $n \geq n_{0}, n_{0}>p /(\ln r-\ln p)$. It results that for a fixed $x_{0}, \varepsilon, \delta$ there exists a natural number $n_{0}=n_{0}\left(x_{0}, \varepsilon, \delta, M_{2}, p, r\right)$ such that for all $n>n_{0}$, we have $S_{2}<\varepsilon / 2$.

Therefore, for all $n>n_{0}$, we have

$$
\left.e^{-r x_{0}} P_{n}\left(\varphi\left(t ; x_{0}\right) ; x_{0}\right)<\varepsilon \quad \text { (i.e., } \lim _{n \rightarrow \infty} e^{-r x_{0}} P_{n}\left(\varphi\left(t ; x_{0}\right) ; x_{0}\right)=0\right) .
$$

It results that $\lim _{n \rightarrow \infty} P_{n}\left(\varphi\left(t ; x_{0}\right) ; x_{0}\right)=0$.

\section{A Voronovskaya-type theorem}

Now we are in the position to state the main result of this paper.

For a fixed $p>0$, let

$$
C_{p}^{2}=\left\{f \in C_{p} \text { such that } f^{\prime}, f^{\prime \prime} \in C_{p}\right\}
$$


6 A Voronovskaya-type theorem for a positive linear operator

Theorem 3.1. If $f \in C_{p}^{2}$, then

$$
\lim _{n \rightarrow \infty} n\left\{P_{n}(f ; x)-f(x)\right\}=\frac{x}{2} f^{\prime \prime}(x)+f^{\prime}(x) \frac{g^{\prime}(1)}{g(1)}
$$

for every fixed $x \in[0, \infty)$.

Proof. We use the Taylor formula for a fixed point $x_{0} \in[0, \infty)$. For all $t \in[0, \infty)$, we have

$$
f(t)=f\left(x_{0}\right)+\left(t-x_{0}\right) f^{\prime}\left(x_{0}\right)+\frac{1}{2}\left(t-x_{0}\right)^{2} f^{\prime \prime}\left(x_{0}\right)+g\left(t ; x_{0}\right)\left(t-x_{0}\right)^{2},
$$

where $g\left(t ; x_{0}\right)$ is the Peano form of the remainder, $g\left(\cdot ; x_{0}\right) \in C_{p}$, and $\lim _{t \rightarrow x_{0}} g\left(t ; x_{0}\right)=0$. Because $P_{n}\left(e_{0} ; x\right)=1$, we can write

$$
\begin{aligned}
P_{n}\left(f ; x_{0}\right)-f\left(x_{0}\right)= & f^{\prime}\left(x_{0}\right) P_{n}\left(t-x_{0} ; x_{0}\right)+\frac{1}{2} f^{\prime \prime}\left(x_{0}\right) P_{n}\left(\left(t-x_{0}\right)^{2} ; x_{0}\right) \\
& +P_{n}\left(g\left(t ; x_{0}\right)\left(t-x_{0}\right)^{2} ; x_{0}\right) .
\end{aligned}
$$

By Cauchy's inequality, we have

$$
P_{n}\left(g\left(t ; x_{0}\right)\left(t-x_{0}\right)^{2} ; x_{0}\right) \leq\left\{P_{n}\left(g^{2}\left(t ; x_{0}\right) ; x_{0}\right)\right\}^{1 / 2}\left\{P_{n}\left(\left(t-x_{0}\right)^{4} ; x_{0}\right)\right\}^{1 / 2} .
$$

The function $\varphi\left(t ; x_{0}\right)=g^{2}\left(t ; x_{0}\right), t \geq 0$, satisfies the conditions of Lemma 2.6; therefore

$$
\lim _{n \rightarrow \infty} P_{n}\left(g^{2}\left(t ; x_{0}\right) ; x_{0}\right)=0
$$

Moreover, by Lemma 2.4, we have

$$
n P_{n}\left(g\left(t ; x_{0}\right)\left(t-x_{0}\right)^{2} ; x_{0}\right) \leq\left\{P_{n}\left(g^{2}\left(t ; x_{0}\right) ; x_{0}\right)\right\}^{1 / 2}\left(n^{2} M_{1}\left(x_{0}\right) \frac{1}{n^{2}}\right)^{1 / 2} .
$$

It results that $\lim _{n \rightarrow \infty} n P_{n}\left(g\left(t ; x_{0}\right)\left(t-x_{0}\right)^{2} ; x_{0}\right)=0$. By the above results and by Lemma 2.3 , we obtain

$$
\lim _{n \rightarrow \infty} n\left(P_{n}\left(f ; x_{0}\right)-f\left(x_{0}\right)\right)=f^{\prime}\left(x_{0}\right) \frac{g^{\prime}(1)}{g(1)}+\frac{x_{0}}{2} f^{\prime \prime}\left(x_{0}\right) .
$$

\section{References}

[1] A. Ciupa, A positive linear operator for approximation in exponential weight spaces, Mathematical Analysis and Approximation Theory, the 5th Romanian-German Seminar on Approximation Theory and Its Applications (RoGer, 2002), Burg, Sibiu, 2002, pp. 85-96.

[2] __ Approximation by a generalized Szasz type operator, Journal of Computational Analysis and Applications 5 (2003), no. 4, 413-424. 
[3] M. Leśniewicz and L. Rempulska, Approximation by some operators of the Szasz-Mirakjan type in exponential weight spaces, Glasnik Matematički. Serija III 32(52) (1997), no. 1, 57-69.

Alexandra Ciupa: Department of Mathematics, Technical University of Cluj-Napoca, 3400 Cluj-Napoca, Romania

E-mail address: ciupa.alexandra@math.utcluj.ro 


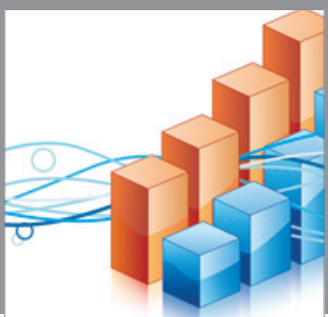

Advances in

Operations Research

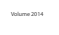

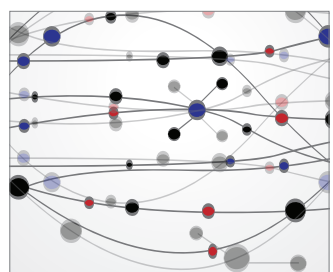

\section{The Scientific} World Journal
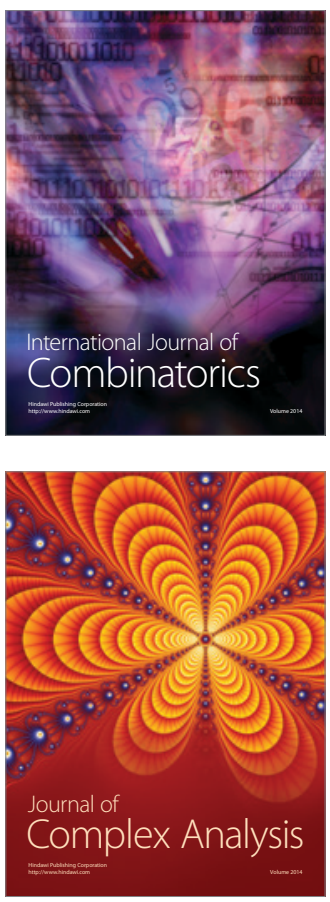

International Journal of

Mathematics and

Mathematical

Sciences
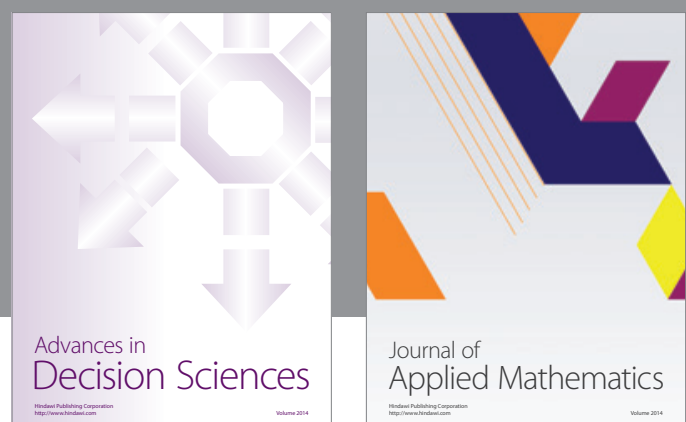

Journal of

Applied Mathematics
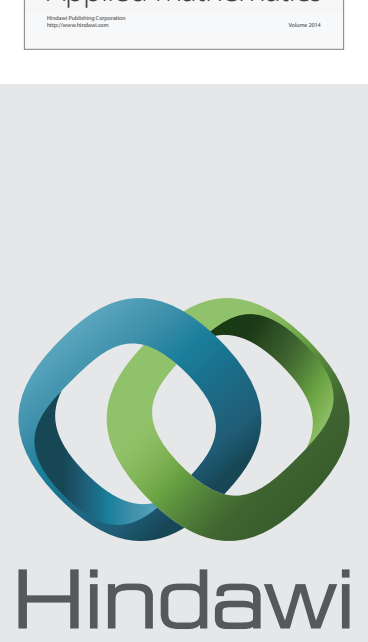

Submit your manuscripts at http://www.hindawi.com
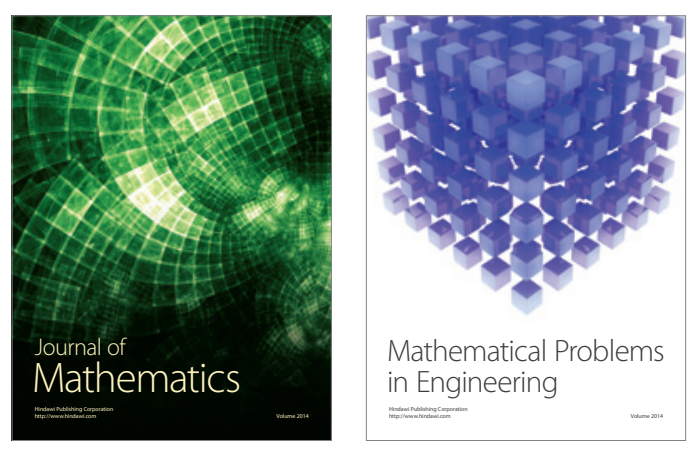

Mathematical Problems in Engineering
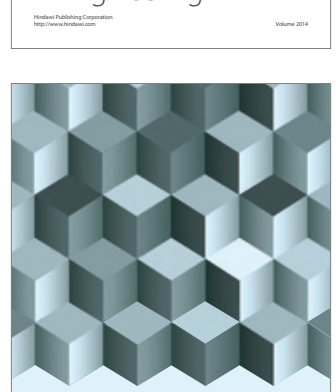

Journal of

Function Spaces
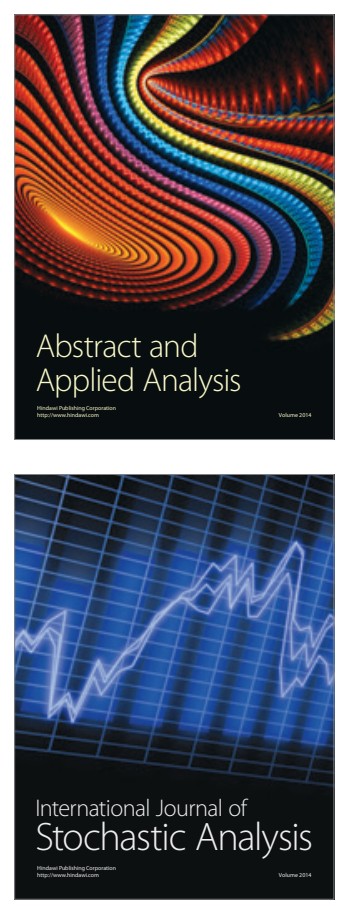

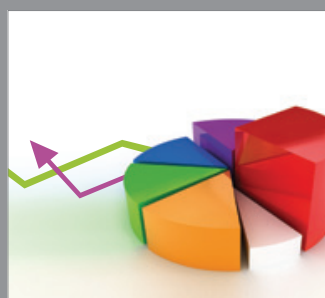

ournal of

Probability and Statistics

Promensencen
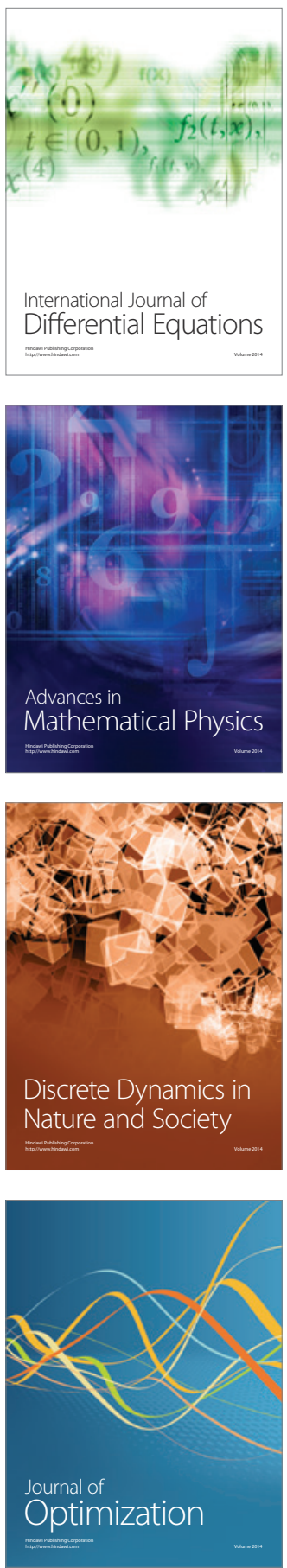